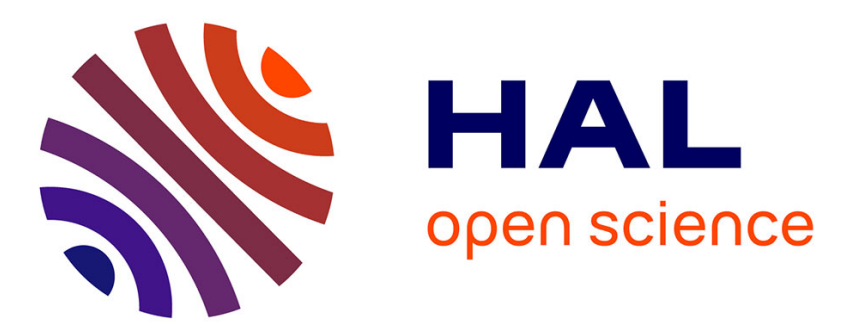

\title{
Velocity Aided Attitude Estimation for Aerial Robotic Vehicles Using Latent Rotation Scaling
}

\author{
Guillaume Allibert, Robert Mahony, Moses Bangura
}

\section{To cite this version:}

Guillaume Allibert, Robert Mahony, Moses Bangura. Velocity Aided Attitude Estimation for Aerial Robotic Vehicles Using Latent Rotation Scaling. IEEE International Conference on Robotics and Automation (ICRA 2016), May 2016, Stockholm, Sweden. hal-01308027

\section{HAL Id: hal-01308027 \\ https://hal.science/hal-01308027}

Submitted on 27 Apr 2016

HAL is a multi-disciplinary open access archive for the deposit and dissemination of scientific research documents, whether they are published or not. The documents may come from teaching and research institutions in France or abroad, or from public or private research centers.
L'archive ouverte pluridisciplinaire HAL, est destinée au dépôt et à la diffusion de documents scientifiques de niveau recherche, publiés ou non, émanant des établissements d'enseignement et de recherche français ou étrangers, des laboratoires publics ou privés. 


\title{
Velocity Aided Attitude Estimation for Aerial Robotic Vehicles Using Latent Rotation Scaling
}

\author{
Guillaume Allibert $^{1}$ and Robert Mahony ${ }^{2,3}$ and Moses Bangura ${ }^{2}$
}

\begin{abstract}
Flight performance of aerial robotic vehicles is critically dependent on the quality of the state estimates provided by onboard sensor systems. The attitude estimation problem has been extensively studied over the last ten years and the development of low complexity, high performance, robust non-linear observers for attitude has been one of the enabling technologies fueling the growth of small scale aerial robotic systems. The velocity aided attitude estimation problem, that is simultaneous estimation of attitude and linear velocity of an aerial platform, has only been tackled using the non-linear observer approach in the last few years. Prior contributions have lead to non-linear observers for which either there is no stability analysis or for which the analysis is extremely complex. In this paper, we propose a simple relaxation of the state space, allowing scaled rotation matrices $R \in \mathbb{R}^{3 \times 3}$ such that $R X^{T}=u I$ where $X=u \hat{R}$ and $u>0$ is a positive scalar, along with additional observer dynamics to force $u \rightarrow 1$ asymptotically. With this simple augmentation of the observer state space, we propose a non-linear observer with a straightforward Lyapunov stability analysis that demonstrates almost global asymptotic convergence along with local exponential convergence. Simulations as well as experimental results are provided to demonstrate the performance of the proposed observer.
\end{abstract}

\section{INTRODUCTION}

Inertial Measurement Units (IMU) are the fundamental sensor systems for estimation of orientation of aerospace vehicles such as spacecrafts, aircrafts and missiles [6]. Most orientation estimators are based on the principle of observing in the body-fixed frame vectorial directions that are known in the inertial frame. For micro-aerial vehicles such as quadrotors, the two most commonly used vectorial measurements are earth's gravity and magnetic fields. In earlier work, Hamel et. al [7] showed that a measurement of the gravitational vector along with angular velocity can be used to estimate roll and pitch angles and gyroscope biases. This approach has been applied extensively in practice for attitude estimation on quadrotor vehicles using accelerometers to estimate gravity, even though in this case the assumptions do not hold exactly [14] due to inertial accelerations of the vehicle. Global Positioning Systems (GPS) can be employed to estimate motion and hence acceleration of the vehicle [9], however, high rate absolute position and velocity measurements are a luxury that most mobile robots operating in urban and indoor environments lack. Recent research into the aerodynamics of quadrotor vehicles have shown that the

\footnotetext{
${ }^{1}$ Guillaume Allibert is with I3S-CNRS, Université de Nice Sophia Antipolis, France. Allibertei3s.unice.fr

${ }^{2}$ Robert Mahony and Moses Bangura are with Research School of Engineering and Information Sciences, Australian National University, Canberra, Australia. \{firstname\}.\{surname\}@anu.edu.au

${ }^{3}$ Also with ARC Centre of Excellence for Robotic Vision.
}

horizontal drag force generated when a quadrotor moves is proportional to the horizontal body-fixed frame velocity [4], [5], [13]. This force corresponds to the horizontal acceleration of the accelerometers and can be measured by the IMU [14]. This measurement can be combined with body mounted gyroscope measurements to obtain an estimate of the horizontal velocity of the quadrotor vehicle [1], [10]. Work by [4] provides the ability to also measure vertical velocity of the airframe and the combination provides a noisy measurement of the body-fixed frame velocity of the vehicle.

In this paper, we consider the problem of designing a nonlinear observer for velocity aided attitude, that is estimating the full body-fixed frame velocity along with the attitude of a quadrotor vehicle. The paper takes a similar approach to the recent paper by the authors [2], by estimating a matrix that is more general than the full rotation matrix in order to simplify the Lyapunov stability analysis. In this paper, only a single degree of freedom is added to the observer state; that is we estimate a scaled rotation $X=u R$ for $R \in S O(3)$ and $u \in \mathbb{R}_{+}$, rather than estimating a rotation in $S O(3)$ directly. It is, of course, trivial to recover the best estimate rotation $\hat{R}=X /\|X\|_{2}$ during evolution of the filter for use in vehicle avionics system. The relaxation of the observer state with a single latent degree of freedom provides the freedom that enables us to undertake a straightforward Lyapunov observer design and stability analysis. The observer is proved to be almost globally exponentially stable; that is the estimate $X(t) \rightarrow R(t)$ converges exponentially to the true attitude in both magnitude and direction for almost all initial conditions. In practice, with perturbations due to signal noise, this leads to global practical exponentially stable. A key benefit of the latent variable dynamics is that the magnitude of the estimate $\|X\|_{2}=u \rightarrow 1$ is known to converge exponentially to unity, and the error $\|X\|_{2}-1$ becomes an excellent measure of the transient convergence of the observer. The resulting observer is low-complexity, robust and can be tuned to have good performance. The performance of the proposed observer is verified by simulation and experimentally on an open-source autopilot [15] controlling a quadrotor vehicle flying in closed loop velocity control in laboratory conditions.

The paper has four sections in addition to the present introduction. In Section II, we introduce the model and some mathematical definitions. Section III presents the proposed observer and provides a stability proof for its performance while Section IV presents some simulation results to verify the performance of the proposed observer and in Section V, we present experimental results of the proposed observer. 


\section{BACKGROUND}

Consider a body-fixed frame denoted $\{B\}$ attached to the vehicle and an inertial frame $\{A\}$ fixed to the ground. A rigid body moving inside the earth's gravity field satisfies [12]

$$
\begin{aligned}
& \dot{V}=-\Omega \times V+g R^{\top} \vec{e}_{3}+a, \\
& \dot{R}=R \Omega_{\times},
\end{aligned}
$$

where $V \in\{B\} \equiv \mathbb{R}^{3}$ denotes the linear velocity of the body-fixed frame $\{B\}$ and $\Omega \in\{B\} \equiv \mathbb{R}^{3}$ denotes the angular velocity of the body frame $\{B\}$ with respect to the inertial frame $\{A\}$ expressed in $\{B\}$. The gravitational acceleration expressed in the inertial frame $\{A\}$ is given by $g \vec{e}_{3}$ where $\vec{e}_{3}=[0 ; 0 ; 1]$ is the unit vector in the $z$-axis. The specific acceleration $a$ is the sum of all non-gravitational forces applied to the body divided by its mass and expressed in the body-fixed frame $\{B\}$. Let $R \in \mathrm{SO}(3)$ denote the rotation matrix representing the orientation of the body-fixed frame $\{B\}$ with respect to the inertial frame $\{A\}$. The linear operator $(.)_{\times}$maps any vector in $\mathbb{R}^{3 \times 1}$ to its corresponding skew-symmetric matrix in $\mathfrak{s o}(3)$ such that $x_{\times} y$ is equal to the cross product $x \times y$ for all $x, y \in \mathbb{R}^{3 \times 1}$.

Assume that the vehicle is equipped with an Inertial Measurement Unit (IMU), which consists of a 3-axis gyroscope, a 3-axis accelerometer and a barometer. The gyroscope provides the measurement of the angular velocity $\Omega$ and the accelerometer measures the specific acceleration $a \in$ $\{B\}$. Additionally, we assume that the linear velocity $V$ of the body-fixed frame $\{B\}$ expressed in the body-fixed frame, can be measured. From a practical point of view, $V_{x}$ and $V_{y}$ measurements can be obtained using accelerometer measurements and a model of aerodynamic drag coefficient [1], [10], [14]. For $V_{z}$, we propose a simple pre-filter based on barometer altitude measurements and accelerometer to estimate $V_{z}$. The estimates of the linear body-fixed frame velocity obtained in this way depend directly on the accelerometer and are extremely noisy. It is crucial to denoise the signals using some observer before the velocity estimate can be used. The experimental section $\S \mathrm{V}$ provides plots of typical measurements and filter outputs that indicate the importance of the role of the observer.

\section{OBSERVER}

In this section, a non-linear observer for estimating the body-fixed frame velocity is proposed. We assume that the full measurement of the body-fixed frame velocity $V \in\{B\}$ is available. If the IMU is also equipped with a magnetometer, then it can be used to provide an additional vector direction measurement directly

$$
m_{B}=R^{\top} m_{A} \in\{B\},
$$

where $m_{A} \in\{A\}$ is the inertial "known" magnetic field. In practice, the magnetic field measurement is often corrupted by onboard magnetic fields and cannot be used for attitude estimation. For this reason, we will initially develop the proposed observer in the case where the magnetic field is not available.
The goal of the observer design is to provide estimates $\hat{V} \in \mathbb{R}^{3}$ and $\hat{R} \in \mathrm{SO}(3)$ of the body-fixed frame velocity and attitude of the vehicle. We will distinguish between the true velocity $\stackrel{\circ}{V}(t)$ and the measured velocity $V(t)$, that is corrupted by noise although the non-linear observer framework does not explicitly model the noise, that is, deterministic stability analysis is based on the relationship $V(t)=\stackrel{\circ}{V}(t)$.

Assumption 1 Assume that the trajectory of the vehicle is sufficiently smooth such that $\Omega(t), \dot{\Omega}(t), \dot{V}(t)$ and $\ddot{V}(t)$ are bounded signals.

Theorem 1 Consider system (1) with $\Omega$, a and $V$ measured and $k_{1}, k_{2}>0$ two positive scalar gains. Consider the observer

$$
\begin{array}{ll}
\dot{\hat{V}}=-\Omega_{\times} \hat{V}+g X^{\top} \vec{e}_{3}+a-k_{1}(\hat{V}-V), \quad \hat{V}(0)=V(0), \\
X=u \hat{R}, \\
\dot{u}=-g k_{2} \tilde{V}^{\top} \hat{R}^{\top} \vec{e}_{3}, \quad u(0)=1, \\
\dot{\hat{R}}=\hat{R} \Omega_{\times}-\frac{g k_{2}}{u}\left(\hat{R} \tilde{V} \times \vec{e}_{3}\right)_{\times} \hat{R}, \quad \hat{R}(0)=I_{3},
\end{array}
$$

where $X$ is thought of as a scaled rotation matrix. Suppose that Assumption 1 is satisfied, then the following properties hold :

1) for almost all initial conditions, the estimate $\hat{V}(t) \rightarrow$ $\stackrel{V}{V}(t)$ and $\hat{R}^{\top} \vec{e}_{3} \rightarrow R^{\top} \vec{e}_{3}$;

2) the equilibrium $\left(\tilde{V}, \tilde{R}^{\top} \vec{e}_{3}\right)=\left(0, \vec{e}_{3}\right)$ is locally exponentially stable.

Proof: Define a velocity error and a rotation error

$$
\begin{gathered}
\tilde{V}=\hat{V}-V \\
\tilde{R}=R \hat{R}^{\top} .
\end{gathered}
$$

The time derivative of $\tilde{V}$ is given by

$$
\dot{\tilde{V}}=-\Omega_{\times} \tilde{V}+(X-R)^{\top} g \vec{e}_{3}-k_{1} \tilde{V} .
$$

Define a candidate Lyapunov function

$$
\mathcal{L}:=\underbrace{\frac{1}{2} \tilde{V}^{\top} \tilde{V}}_{\mathcal{L}_{A}}+\underbrace{\frac{1}{2 k_{2}}\left\|(X-R)^{\top} \vec{e}_{3}\right\|^{2}}_{\mathcal{L}_{B}} .
$$

Using (2a), the time derivative of $\mathcal{L}_{A}$ is

$$
\dot{\mathcal{L}}_{A}=g \tilde{V}^{\top}(X-R)^{\top} \vec{e}_{3}-k_{1} \tilde{V}^{\top} \tilde{V} .
$$

Define $y:=(X-R)^{\top} \vec{e}_{3}$ and $\Delta=-\frac{g k_{2}}{u}\left(\hat{R} \tilde{V} \times \vec{e}_{3}\right) \in \mathbb{R}^{3}$, one has

$$
\begin{aligned}
\dot{y} & =\left(\dot{u} \hat{R}+u \dot{\hat{R}}-R \Omega_{\times}\right)^{\top} \vec{e}_{3}, \\
& =\left(\dot{u} \hat{R}+u \Delta_{\times} \hat{R}+(X-R) \Omega_{\times}\right)^{\top} \vec{e}_{3}, \\
& =\left(\dot{u} \hat{R}^{\top}+u \hat{R}^{\top} \Delta_{\times}^{\top}\right) \vec{e}_{3}-\Omega_{\times} y .
\end{aligned}
$$

From here, the derivative of $\mathcal{L}_{B}$ along trajectories of the system is given by

$$
\begin{aligned}
\dot{\mathcal{L}}_{B} & =\frac{1}{k_{2}} y^{\top} \dot{y}, \\
& =\frac{1}{k_{2}}\left((X-R)^{\top} \vec{e}_{3}\right)^{\top}\left(\dot{u} \hat{R}^{\top}+u \hat{R}^{\top} \Delta_{\times}^{\top}\right) \vec{e}_{3}, \\
& =\frac{1}{k_{2}}\left(u \vec{e}_{3}^{\top} \Delta_{\times} \hat{R}+\dot{u} \vec{e}_{3}^{\top} \hat{R}\right) y .
\end{aligned}
$$


Consequently, the time derivative of $\mathcal{L}$ becomes

$$
\begin{aligned}
\dot{\mathcal{L}} & =g \tilde{V}^{\top}(X-R)^{\top} \vec{e}_{3}+\frac{1}{k_{2}}\left(u \vec{e}_{3}^{\top} \Delta_{\times} \hat{R}+\dot{u} \vec{e}_{3}^{\top} \hat{R}\right) y-k_{1} \tilde{V}^{\top} \tilde{V}, \\
& =\frac{1}{k_{2}}\left(g k_{2} \tilde{V}^{\top} \hat{R}^{\top}+u \vec{e}_{3}^{\top} \Delta_{\times}+\dot{u} \vec{e}_{3}^{\top}\right) \hat{R} y-k_{1} \tilde{V}^{\top} \tilde{V} .
\end{aligned}
$$

Recall the expressions for $\dot{u}$ and $\Delta$ and note that

$$
u \Delta_{\times}^{\top} \vec{e}_{3}+\dot{u} \vec{e}_{3}=-g k_{2} \hat{R} \tilde{V} .
$$

From this, it is straightforward to see that

$$
\dot{\mathcal{L}}=-k_{1} \tilde{V}^{\top} \tilde{V} \leq 0
$$

Since the time derivative of $\dot{\mathcal{L}}$ is semi-negative definite and $\mathcal{L}$ is positive definite, then $\tilde{V}$ and $X$ are bounded. In view of (3) and Assumption 1, one deduces that $\dot{\tilde{V}}$ is bounded and it follows that $\ddot{\mathcal{L}}$ is also bounded. This is sufficient to ensure that $\dot{\mathcal{L}}$ is uniformly continuous along trajectories of the system. Applying Barbalat's lemma ensures the convergence of $\dot{\mathcal{L}} \rightarrow 0$ and the convergence of $\tilde{V}$ to 0 follows.

The same procedure is performed to prove that $\ddot{\tilde{V}}$ is bounded (since $\dot{\Omega}, \dot{\tilde{V}}, \dot{X}$ and $\dot{\hat{R}}$ are bounded) and consequently to demonstrate the uniform continuity of $\dot{\tilde{V}}$. Barbalat's lemma ensures the convergence of $\dot{\tilde{V}}$ to 0 . This in turn implies, from (3), the convergence of $g X^{\top} \vec{e}_{3}$ to $g R^{\top} \vec{e}_{3}$. Finally, substituting $X=u \hat{R}$, one sees that $u \hat{R}^{\top} \vec{e}_{3} \rightarrow$ $R^{\top} \vec{e}_{3}$. Taking norms, one has $\left|u \vec{e}_{3}\right| \rightarrow 1$, that is $u$ converges to unity. It follows that $\hat{R}^{\top} \vec{e}_{3} \rightarrow R^{\top} \vec{e}_{3}$ by continuity and consequently, $\tilde{R}^{\top} \vec{e}_{3} \rightarrow \vec{e}_{3}$.

We go on to prove Property 2. Close to the equilibrium points $\left(\tilde{V}, \tilde{R}^{\top} \vec{e}_{3}\right)=\left(0, \vec{e}_{3}\right)$, one can write

$$
\begin{aligned}
\tilde{V} & \approx 0+\delta \tilde{V}, \\
u & \approx 1+\delta u, \\
\hat{R} & \approx\left(\begin{array}{ccc}
1 & -\delta_{\psi} & \delta_{\theta} \\
\delta_{\psi} & 1 & -\delta_{\phi} \\
-\delta_{\theta} & \delta_{\phi} & 1
\end{array}\right) .
\end{aligned}
$$

From $\Delta, \dot{u}$ and the previous equations, one has

$$
\begin{aligned}
& \Delta=g k_{2}\left(\begin{array}{c}
-\delta \tilde{V}_{y} \\
\delta \tilde{V}_{x} \\
0
\end{array}\right), \\
& \dot{u}=-g k_{2} \delta \tilde{V}_{z},
\end{aligned}
$$

and consequently, $\dot{\tilde{V}}=-\Omega_{\times} \tilde{V}+(X-R)^{\top} g \vec{e}_{3}-k_{1} \tilde{V}$ verifies

$$
\begin{aligned}
& \delta \dot{\tilde{V}}=-\Omega \times \delta \tilde{V}+g(X-R)^{\top} \vec{e}_{3}-k_{1} \delta \tilde{V}, \\
& =-\Omega \times \delta \tilde{V}+g\left(u \hat{R}^{\top} R-I\right) R^{\top} \vec{e}_{3}-k_{1} \delta \tilde{V}, \\
& =\left(\begin{array}{ccc}
-k_{1} & \Omega_{3} & -\Omega_{2} \\
-\Omega_{3} & -k_{1} & \Omega_{1} \\
\Omega_{2} & -\Omega_{1} & -k_{1}
\end{array}\right) \delta \tilde{V}+g\left(\begin{array}{c}
-\delta_{\theta} \\
\delta_{\phi} \\
\delta u
\end{array}\right) \text {. }
\end{aligned}
$$

If we note $\xi_{1}=\left[\delta \tilde{V}_{x} ; \delta \tilde{V}_{y} ; \delta \tilde{V}_{z}\right]^{\top}$ and $\xi_{2}=\left[-\delta_{\theta} ; \delta_{\phi} ; \delta u\right]^{\top}$, the previous equation can be written as

$$
\dot{\xi}_{1}=\left(-k_{1} I_{3}-\Omega_{\times}\right) \xi_{1}+g \xi_{2} .
$$

From previous equations, it is straightforward to verify that

$$
\begin{aligned}
\dot{\xi}_{2} & =-g k_{2}\left(\begin{array}{l}
\delta \tilde{V}_{x} \\
\delta \tilde{V}_{y} \\
\delta \tilde{V}_{z}
\end{array}\right), \\
& =-g k_{2} \xi_{1} .
\end{aligned}
$$

We can conclude from (9) and (10) that the linearised system of (2) is given by

$$
\dot{\xi}=A(t) \xi,
$$

with

$$
A(t)=\left(\begin{array}{cccccc}
-k_{1} & \Omega_{3} & -\Omega_{2} & g & 0 & 0 \\
-\Omega_{3} & -k_{1} & \Omega_{1} & 0 & g & 0 \\
\Omega_{2} & -\Omega_{1} & -k_{1} & 0 & 0 & g \\
-g k_{2} & 0 & 0 & 0 & 0 & 0 \\
0 & -g k_{2} & 0 & 0 & 0 & 0 \\
0 & 0 & -g k_{2} & 0 & 0 & 0
\end{array}\right),
$$

and $\xi=\left[\xi_{1} ; \xi_{2}\right]$. From here, we have to prove that the origin of the linear time varying system given by (11) is uniformly exponentially stable. The following proof is based on the results obtained in [11, Theorem 1], which establish sufficient conditions for exponential stability of linear time varying system having the form

$$
\left(\begin{array}{c}
\dot{\mathbf{x}} \\
\dot{\mathbf{y}}
\end{array}\right)=\left(\begin{array}{cc}
\mathcal{A}(t) & \mathcal{B}^{\top}(t) \\
-\mathcal{C}(t) & 0
\end{array}\right)\left(\begin{array}{l}
\mathbf{x} \\
\mathbf{y}
\end{array}\right)
$$

which corresponds to (11). By identification, one obtains

$$
\mathcal{A}(t)=-k_{1} I_{3 \times 3}-\Omega_{\times}, \mathcal{B}(t)=g I_{3 \times 3}, \mathcal{C}(t)=g k_{2} I_{3 \times 3} .
$$

We have to verify the two assumptions of Theorem 1 of [11]. The first one is easily verified since $|\mathcal{B}|$ and $\left|\frac{\partial \mathcal{B}}{\partial t}\right|$ remain bounded. The second assumption is also satisfied since $\mathcal{P}=k_{2} I_{3 \times 3}$ and $\mathcal{Q}=2 k_{1} k_{2} I_{3 \times 3}$ are symmetric, constant and positive definite matrices satisfying the required relations given by $\mathcal{P} \mathcal{B}^{\top}=\mathcal{C}^{\top}$ and $-\mathcal{Q}=\mathcal{A}^{\top} \mathcal{P}+\mathcal{P} \mathcal{A}+\dot{\mathcal{P}}$. Finally, it remains to prove that $\mathcal{B}$ is uniformly persistently exciting which can be verified for any positive number $\mu$ and $T>\frac{\mu}{g^{2}}$ since for all time $t>0$, one has

$$
\int_{t}^{T+t} \mathcal{B}(\tau) \mathcal{B}^{\top}(\tau) d \tau=g^{2} T I_{3 \times 3}>\mu I_{3 \times 3} .
$$

From here, the application of Theorem 1 of [11] ensures the uniform exponential stability of the origin of (11). This concludes the proof.

Consider now the case where the IMU is equipped with a magnetometer. In the same spirit of [8] and under assumption 1 , the second result of the paper is stated.

Theorem 2 Consider the observer in Theorem (1) under the same assumptions. The dynamics of $\hat{R}$ is now given by

$$
\begin{aligned}
\dot{\hat{R}}= & \hat{R} \Omega_{\times}-\frac{g k_{2}}{u}\left(\hat{R} \tilde{V} \times \vec{e}_{3}\right)_{\times} \hat{R}+ \\
& k_{3} \hat{R}\left(\left(\left(m_{B} \times \hat{m}_{B}\right)^{\top} X^{\top} \vec{e}_{3}\right) X^{\top} \vec{e}_{3}\right)_{\times}, \hat{R}(0)=I_{3},
\end{aligned}
$$

with $\hat{m}_{B}=\hat{R}^{\top} m_{B}$ and $k_{3}$ a positive scalar. Then, the following property holds:

1) for almost all initial conditions, the estimate $\hat{V}(t) \rightarrow$ $\stackrel{\circ}{V}(t)$ and $\hat{R}^{\top} \vec{e}_{3} \rightarrow R^{\top} \vec{e}_{3}$

2) the equilibrium $(\tilde{V}, \tilde{R})=(0, I)$ is locally exponentially stable and almost globally asymptotically stable. Thus, 
for almost all initial conditions $(\hat{V}(0), \hat{R}(0))$, the trajectory $(\hat{V}(t), \hat{R}(t))$ converges to the system trajectory $(\stackrel{\circ}{V}(t), R(t))$.

Proof: Consider the same candidate Lyapunov function as (4). The time derivative of the term $\mathcal{L}_{A}$ does not change. Define $\Psi=\left(\left(\left(m_{B} \times \hat{m}_{B}\right)^{\top} X^{\top} \vec{e}_{3}\right) X^{\top} \vec{e}_{3}\right) \in \mathbb{R}^{3}$, the time derivative of the term $\mathcal{L}_{B}$ becomes

$$
\begin{aligned}
\dot{\mathcal{L}}_{B} & =\frac{1}{k_{2}} y^{\top} \dot{y}, \\
& =\frac{1}{k_{2}}(u \vec{e}_{3}^{\top} \Delta_{\times} \hat{R}+\dot{u} \vec{e}_{3}^{\top} \hat{R}+\underbrace{u \vec{e}_{3}^{\top} \hat{R} \Psi_{\times}}_{T_{3}}) y,
\end{aligned}
$$

where the term $T_{3}$ is the only change on (6). Replacing $\Psi$ by its definition in the previous equation, one obtains

$$
\begin{aligned}
T_{3} & =\frac{1}{k_{2}} \vec{e}_{3}^{\top} \underbrace{u \hat{R}}_{X} \Psi_{\times} y, \\
& =\frac{1}{k_{2}} \vec{e}_{3}^{\top} X(\underbrace{\left(\left(m_{B} \times \hat{m}_{B}\right)^{\top} X^{\top} \vec{e}_{3}\right)}_{\alpha} X^{\top} \vec{e}_{3})_{\times} y, \\
& =\frac{\alpha}{k_{2}} \underbrace{\vec{e}_{3}^{\top} X\left(X^{\top} \vec{e}_{3}\right)_{\times}}_{=0} y .
\end{aligned}
$$

We conclude that the term $T_{3}$ is equal to zero and consequently, the innovation term $\Psi$ does not perturb the previous results obtained in Theorem 1.

We now prove Property 2 . The time derivative of $\tilde{R}$ satisfies

$$
\begin{aligned}
\dot{\tilde{R}} & =\dot{R} \hat{R}^{\top}+R \dot{\hat{R}^{\top}}, \\
& =\tilde{R} \Delta_{\times}^{\top}+R \Psi_{\times}^{\top} R^{\top} \tilde{R} .
\end{aligned}
$$

As a result of Proposition 1, one ensures the convergence of $\tilde{V}$ to $0, \hat{R}^{\top} \vec{e}_{3} \rightarrow R^{\top} \vec{e}_{3}$ and $u$ to unity. Consequently, we deduce that $\tilde{R} \vec{e}_{3} \rightarrow \vec{e}_{3}$. It follows that the zero dynamics of $\tilde{R}$ converges to

$$
\begin{aligned}
\dot{\tilde{R}} & =\tilde{R} \Delta_{\times}^{\top}+R \Psi_{\times}^{\top} R^{\top} \tilde{R}, \\
& \rightarrow R \Psi_{\times}^{\top} R^{\top} \tilde{R}, \\
& \rightarrow-k_{3} R\left(\left(\left(m_{B} \times \hat{m}_{B}\right)^{\top} X^{\top} \vec{e}_{3}\right) X^{\top} \vec{e}_{3}\right)_{\times} R^{\top} \tilde{R}, \\
& \rightarrow-k_{3} u^{2}\left(\left(\left(m_{A} \times \tilde{R} m_{A}\right)^{\top} \tilde{R} \vec{e}_{3}\right) \tilde{R} \vec{e}_{3}\right)_{\times} \tilde{R}, \\
& \rightarrow-k_{3}\left(\left(\left(m_{A} \times \tilde{R} m_{A}\right)^{\top} \vec{e}_{3}\right) \vec{e}_{3}\right)_{\times} \tilde{R} .
\end{aligned}
$$

Close to the equilibrium $\tilde{R}=I$, we write $\tilde{R}$ as

$$
\tilde{R} \approx(I+\delta \tilde{R})=\left(\begin{array}{ccc}
1 & -\delta_{\tilde{\psi}} & 0 \\
\delta_{\tilde{\psi}} & 1 & 0 \\
0 & 0 & 1
\end{array}\right),
$$

since $\tilde{R} \vec{e}_{3} \rightarrow \vec{e}_{3}$ and the zero dynamics (16) satisfies

$$
\operatorname{vex}(\dot{\tilde{R}})=\left(\begin{array}{c}
0 \\
0 \\
\dot{\delta}_{\tilde{\psi}}
\end{array}\right)=-\left(\begin{array}{c}
0 \\
0 \\
k_{3}\left(m_{A 1}^{2}+m_{A 2}^{2}\right) \delta_{\tilde{\psi}}
\end{array}\right),
$$

where $m_{A 1}$ and $m_{A 2}$ are the two first entries of $m_{A}$ and vex is the inverse of the $(\cdot)_{\times}$operator that maps a skew matrix to its associated vector velocity representation. This last result clearly indicates the local exponential stability of the equilibrium. This concludes the proof.
Remark 1 The innovation term $\Psi$ is chosen orthogonal to $\hat{R} \vec{e}_{3}$ in order to obtain global decoupling of roll and pitch estimation from magnetometer measurements.

Remark 2 It is important to note that in both observers, the scale factor u converges to unity. This scale factor can be viewed as performance criterion of the observer. As one can see in the simulation section, in the presence of perturbations or unmodelled effects, the value of $u$ can deviate from unity. When the velocity estimates are used in a control scheme, the scale factor can be used as a measure to switch to another control loop in order to avoid bad control performances or a crash in the worst case scenario. Further results are shown in the experimental section where initially as the filter converges $\hat{V}=\stackrel{V}{V}$ so too is $u \rightarrow 1$. During this time, the filter results are suboptimal.

\section{Simulation}

In this section, we illustrate through simulation results the observer proposed in Theorem 1. Results highlight the performance of the proposed observer but also a measure of performance of the filter can be made based on the value of $u$. Simulations are performed for a model of the quadrotor aerial vehicle used in the experimental section. For the presented simulation, initial conditions are chosen such that the initial error variables satisfy $\hat{V}(0)=[0 ; 0 ; 0]$, $u(0)=1$ and $\hat{R}(0)=I_{3 \times 3}$.

The time evolution of the body velocity estimation errors as well as the scale $u$ are plotted. Partial rotation errors given by $\left((\hat{R}-R)^{\top} \vec{e}_{3}\right)^{\top} \vec{e}_{i}$ for $i \in(1,2,3)$ where $\vec{e}_{1}=$ $[1 ; 0 ; 0]$ and $\vec{e}_{2}=[0 ; 1 ; 0]$ are also given. From results (see Figure 1), one observes that convergence is obtained for all variables with good convergence rates.

In order to show that the scale $u$ can be used as a performance criteria of the filter, we add on each velocity estimate a constant step error between 2 and 3 seconds of the simulation. As expected, these perturbations are quickly rejected by the filter. One can see that the $u$ value deviates from 1 at two instances during this time interval thus clearly indicating that the velocity estimation is not optimal at these times.
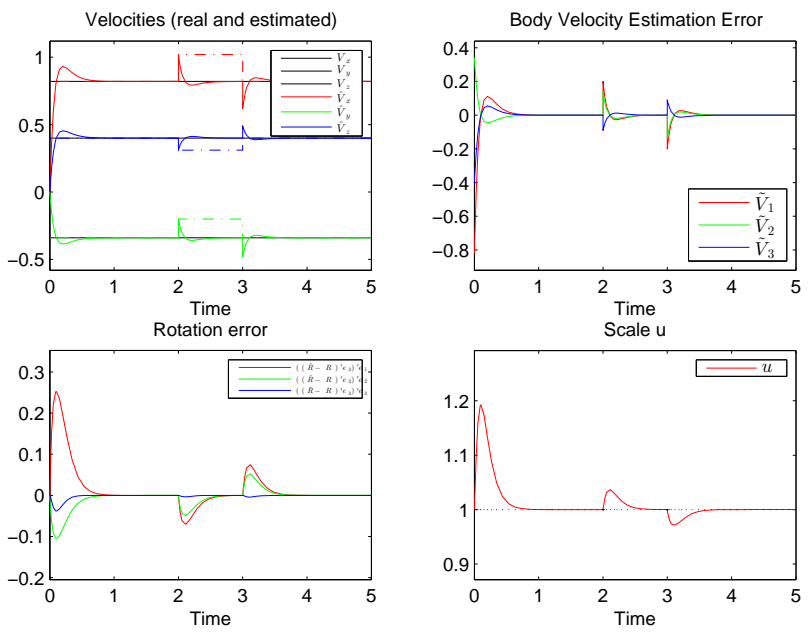

Fig. 1: Simulation results with perturbations at time between 2 and $3 \mathrm{~s}$. 


\section{EXPERIMENTATION}

In this section we demonstrate the performance of the velocity aided filter (Theorem 1) by using it on a quadrotor platform in velocity control mode. The quadrotor uses the 32bit open-source autopilot, PX4 [15] on which the filter was implemented. The section describes how the body-fixed frame velocity measurements can be obtained onbard the autopilot and the resulting filter output evaluated against ground truth obtained from a Vicon motion capture system [16] to demonstrate the performance of the filter.

\section{A. Body fixed-frame velocity measurements}

If the accelerometer measurement is denoted $\bar{a}, T \in \mathbb{R}$ is the total exogenous force produced by the rotors, $D \in \mathbb{R}^{3}$ is the drag force in $\{B\}$, then (1a) can be rewritten as

$$
\dot{V}=-\Omega \times V+g R^{\top \vec{e}_{3}}-\frac{T}{m} \vec{e}_{3}-\frac{D}{m},
$$

knowing that the accelerometer measures external forces,

$$
\begin{aligned}
\bar{a} & =-\frac{T}{m} \vec{e}_{3}-\frac{D}{m}, \\
& =-\frac{1}{m}\left(\begin{array}{l}
D \\
T
\end{array}\right) .
\end{aligned}
$$

Given that $D^{\top} \vec{e}_{3}=0$ [2] and the drag force is expressed as

$$
D=-T\left(\begin{array}{ccc}
\bar{c} & 0 & 0 \\
0 & \bar{c} & 0 \\
0 & 0 & 0
\end{array}\right) V
$$

where $\bar{c}>0$ is the drag coefficient.

Using $T=m \bar{a}^{\top} \vec{e}_{3}$, the following measurement equations are obtained

$$
\bar{a}_{x}=\bar{a}_{z} \bar{c} \bar{V}_{x}, \quad \bar{a}_{y}=\bar{a}_{z} \bar{c} \bar{V}_{y}
$$

where $\bar{V}_{x}, \bar{V}_{y}$ represent the measurements of the translational linear velocities. To obtain the velocity measurement in the vertical direction $\left(\bar{V}_{z}\right)$, [4], [2] proposed the concept of using aerodynamic power. Given that the PX4 is fitted with a barometer, we therefore do not use the aerodynamic power approach. If the barometer measures altitude $\bar{z}, \hat{z}$ is an estimate of the height from these measurements and $v \in \mathbb{R}^{3}$ is the velocity of the vehicle in the inertial frame, $\bar{v}_{z m}$ are pseudo velocity measurements, then we propose the following complementary filter for the estimation of $\bar{v}_{z}$, the measured vehicle velocity in $\vec{e}_{3}$ in inertial frame

$$
\begin{aligned}
\dot{\hat{z}} & =\bar{v}_{m z}-k_{4}(\hat{z}-\bar{z}), \\
\dot{\bar{v}}_{m z} & =\vec{e}_{3}^{\top}(\hat{R} \bar{a})+g-\beta^{a z}-k_{5}(\hat{z}-\bar{z}), \\
\dot{\beta}^{a z} & =k_{6}(\hat{z}-\bar{z}),
\end{aligned}
$$

where $k_{4}, k_{5}, k_{6}$ are positive scalar gains and $\beta^{a z} \in \mathbb{R}$ is an estimate of the bias. From a flight test, results comparing the barometer measured heights $(\bar{z})$ to the estimated heights $(\hat{z})$ and ground truth Vicon height measurements are shown in Figure 2. The results also show the obtained estimate of the vertical velocity from the barometer measurements compared to that obtained using Vicon. These results show the validity of the estimated vertical velocity of the filter (18) to ground truth Vicon measurements. Hence with the obtained $\dot{\hat{z}}$ from the barometer, the measurement $\bar{v}_{z}=\dot{\hat{z}}$ is obtained.

Assuming that the attitude of the vehicle is such that $\hat{R}=$ $R$, then with $\bar{v}_{z}, \bar{V}_{x}$ and $\bar{V}_{y}$ known, the simple algebraic relationship $\bar{v}=\hat{R} \bar{V}$ is used to obtain $\bar{V}_{z}$. Hence with all elements of $\bar{V}=\left(\bar{V}_{x}, \bar{V}_{y}, \bar{V}_{z}\right)^{\top}$ known, they are then used as measurements to the filter proposed in Theorem 1.
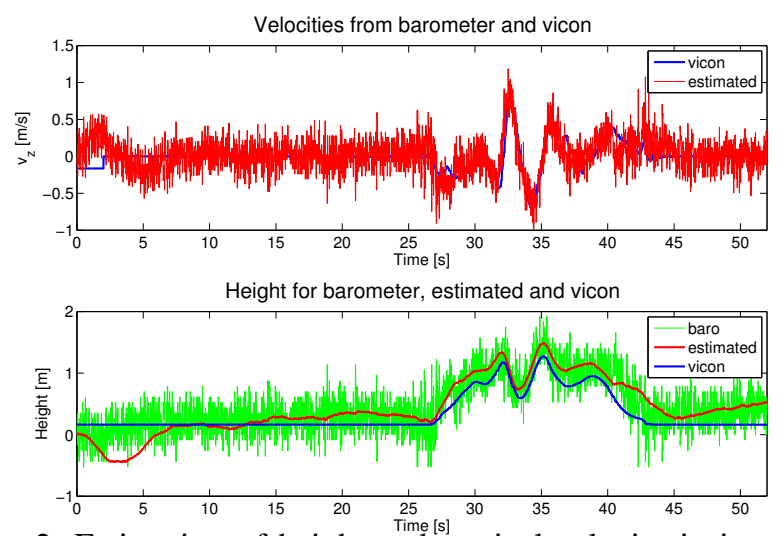

Fig. 2: Estimation of height and vertical velocity in inertial frame using onboard barometric sensor. The results show that the computed estimated velocity (red) matches the ground truth Vicon velocity (blue) measurements despite a slight drift in barometer height measurements with time as indicated by the raw barometer (green) and filtered/estimated (red) measurements compared to Vicon measurements (blue).

\section{B. Experimental results}

In order to compare the estimated attitude to ground truth measurements obtained from Vicon, one needs to ensure that the $\vec{e}_{1}$ direction of the onboard filter aligns with the $\vec{e}_{1}$ direction from Vicon attitude measurements. Hence, we propose the following additional innovation term for the filter equation (12a)

$$
\Delta_{v}=-k_{2}^{v i c o n} \hat{R}\left(\vec{e}_{1} \times \hat{R} \hat{R}_{v}^{\top} \vec{e}_{1}\right)_{\times} \hat{R},
$$

where $R_{v}$ is the attitude measured by Vicon and $k_{2}^{v i c o n} \in$ $\mathbb{R}^{3}$ is a positive definite gain matrix. Flying the quadrotor in closed loop velocity control mode with the controller proposed in [3] and using a mobile phone to set the desired translational velocties while we manually control the force in the vertical direction, the results for the estimated attitude and velocities are shown in Figure 3.

From these results and looking at the velocities, it is clearly evident that the observer achieved its intended purpose of smoothening out the noise despite the data recorded at half the operating frequency of the filter $(200 \mathrm{~Hz})$. Looking at Figure 4, the latent scaling factor $u$, it is easily seen that $u \rightarrow 1$ after 20 s hence after this time the estimation is optimal and therefore the filtered velocities can be used in closed loop velocity control with $u \approx 1, \forall t>20$. As seen in the high level of noise at this point, the vehicle is armed and then flown. Figure 3 also shows a comparison of the resulting estimated attitude to ground truth Vicon measurements in Euler angles. The results show the equivalence of the estimated to ground truth Vicon attitude measurements. It is worth 

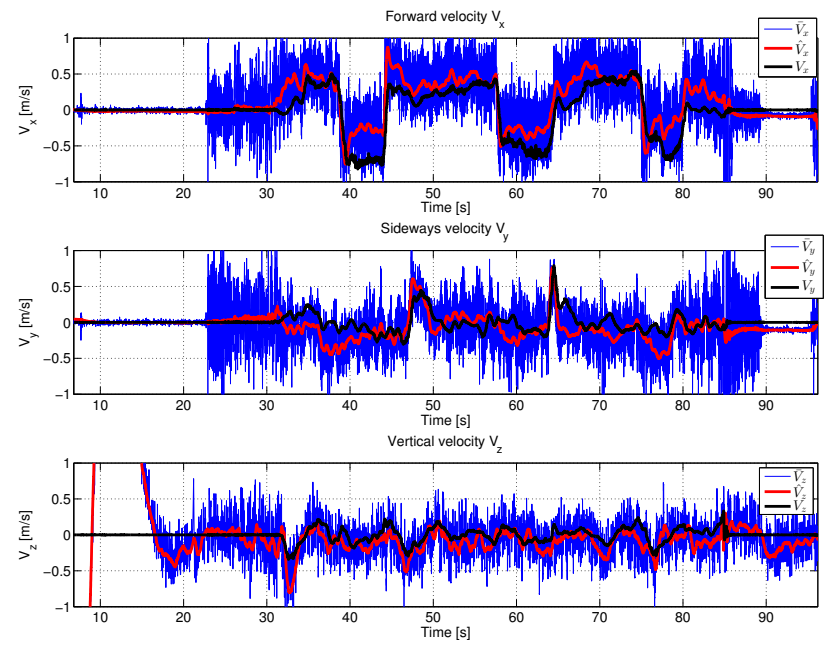

Roll attitude $\phi$
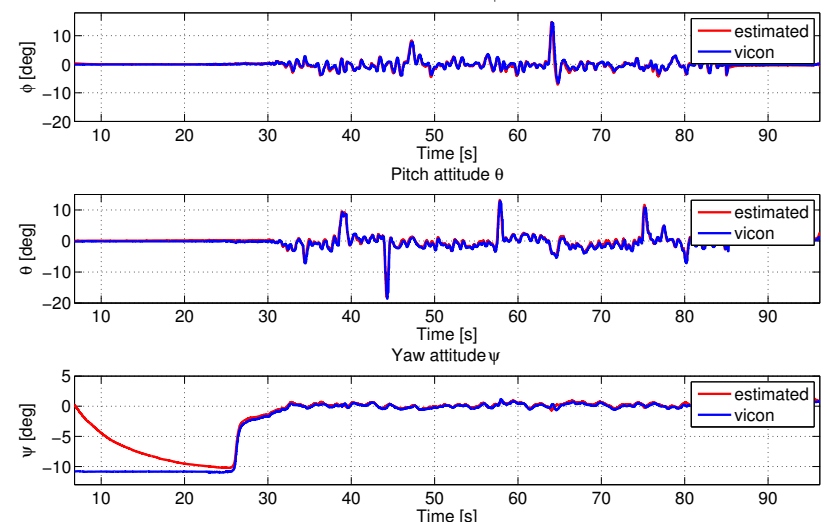

Fig. 3: Results of the output of the filter. In the first set of plots, we show the output of measured $\bar{V}$ (blue) to estimated $\hat{V}$ (red) and ground truth (black) linear velocities with data recorded onboard the autopilot. The resulting attitude from the flight is also shown and compared to Vicon ground truth.

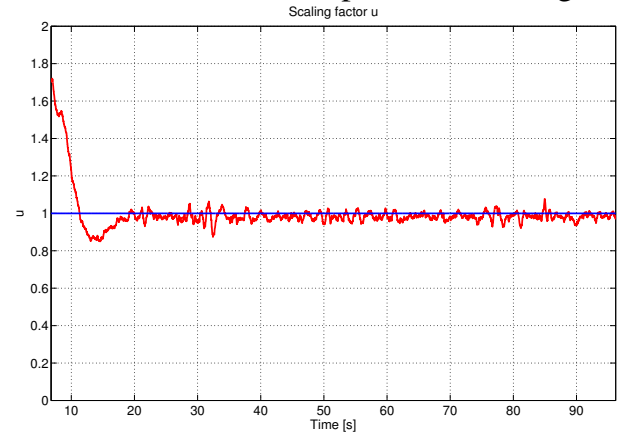

Fig. 4: Convergence of the latent scaling factor $u \rightarrow 1$.

noting that the onboard estimated yaw using magnetometer measurements aligns with the measured Vicon attitude. This is as a result of the innovation term (19).

\section{CONCLUSION}

In this paper, the problem of designing a non-linear observer for velocity aided attitude, that is estimating the full body-fixed frame velocity along with the attitude of quadrotor vehicles is presented. The proposed observer is low-complexity, robust and can be tuned easily to have good performance. Rigorous stability analysis based on Lyapunov theory demonstrates almost global asymptotic convergence along with local exponential convergence. The originality of the proposed approach lies in adding only one degree of freedom into the observer state. Moreover, a performance criterion, very useful when velocity estimates are used in control loop can be obtained easily using the value of the scale $u$. Simulation results are presented to provide a clear picture of the performance of the proposed observer. Experimental results also show that the proposed scheme is effective even when the input to the observer is extremely noisy and can be used in closed loop velocity control.

\section{ACKNOWLEDGMENT}

The authors would like to thank Tarek Hamel (I3S-CNRS, UNS) and Minh Duc Hua (ISIR-CNRS) for the help and guidance. This research was supported by the ANR-Equipex project "Robotex", the ANR SCAR and the Australian Research Council through Discovery Grant DP120100316 "Integrated High-Performance Control of Aerial Robots in Dynamic Environments".

\section{REFERENCES}

[1] D. Abeywardena, S. Kodagoda, G. Dissanayake, and R. Munasinghe. Improved state estimation in quadrotors mavs. Robotics Automation Magazine, IEEE, 20(4):32 - 39, 2013.

[2] G. Allibert, D. Abeywardena, M. Bangura, and R. Mahnoy. Estimating body-fixed frame velocity and attitude from inertial measurements for a quadrotor vehicle. In IEEE Multi-conference on Systems and Control (MSC), pages 978-983, 2014.

[3] M. Bangura, F. Kuipers, G. Allibert, and R. Mahony. Non-linear velocity aided attitude estimation and velocity control for quadrotors. In in Proc. Australasian Conf. Robotics Automation, 2015.

[4] M. Bangura, H. Lim, H.J. Kim, and R. Mahony. Aerodynamic power control for multirotor aerial vehicles. In in Proc. IEEE Int. Conf. Robotics Automation, pages 529-536, 2014.

[5] M. Bangura and R. Mahony. Nonlinear dynamic modeling for high performance control of a quadrotor. In in Proc. Australasian Conf. Robotics Automation, pages 3-5, 2012.

[6] J.L. Crassidis, F.L. Markley, and Y. Cheng. Survey of nonlinear attitude estimation methods. Journal of Guidance, Control, and Dynamics, 30(1):12-28, 2007.

[7] T. Hamel and R. Mahony. Attitude estimation on so(3) based on direct inertial measurements. In in Proc. IEEE Int. Conf. Robotics Automation, pages 2170-2175, 2006.

[8] M-D. Hua, P. Martin, and T. Hamel. Velocity-aided attitude estimation for accelerated rigid bodies. In IEEE Conf. on Decision and Control $(C D C)$, pages 328-333, 2014.

[9] M.D. Hua. Attitude estimation for accelerated vehicles using gps/ins measurements. Control Engineering Practice (Special Issue on Aerial Robotics), 18(7):723 - 732, 2010.

[10] R.C. Leishman, J.C. Macdonald JR., R.W. Beard, and T.W. Mclain. Quadrotors and accelerometers: state estimation with an improved dynamic model. Control Systems Magazine, IEEE, 34(1):28 - 41, 2014.

[11] A. Loria and E. Panteley. Uniform exponential stability of linear timevarying systems: revisited. Systems \& Control Letters, 47(1):13-24, 2002.

[12] R. Mahony, T. Hamel, and J.-M. Pflimlin. Nonlinear complementary filters on the special orthogonal group. IEEE Trans. Autom. Contr., 53(5):1203-1218, 2008.

[13] R. Mahony, V. Kumar, and P. Corke. Multirotor aerial vehicles: Modeling, estimation, and control of quadrotor. Robotics Automation Magazine, IEEE, 19(3):20-32, 2012.

[14] P. Martin and E. Salaun. The true role of accelerometer feedback in quadrotor control. In in Proc. IEEE Int. Conf. Robotics Automation, pages 1623-1629, 2010.

[15] L. Meier, D. Honegger, and M. Pollefeys. Px4: A node-based multithreaded open source robotics framework for deeply embedded platforms. In in Proc. IEEE Int. Conf. Robotics Automation, pages 6235 - 6240, 2015.

[16] Vicon Team. Vicon homepage. http://www.vicon.com/, 2015. [Online; 15-07-2015]. 